\title{
Georg Simmel: Philosophie des Geldes
}

\author{
Simmel, Georg (geb. 1.3.1858 Berlin, gest. 26.9.1918 Straßburg) \\ Philosophie des Geldes \\ EA: Leipzig: Duncker \& Humblot 1900 \\ VA: Frankfurt am Main: Suhrkamp 1989 \\ (= Georg Simmel Gesamtausgabe, Bd. 6)
}

Die Philosophie des Geldes stellt das wirkungsgeschichtlich bedeutendste Werk Simmels dar. Obgleich er sie bewußt als ein philosophisches Buch konzipiert hat, ist ihr doch auch innerhalb der Fachgeschichte der Soziologie immer wieder der Rang eines soziologischen Hauptwerkes zugesprochen worden. Dies liegt zum einen daran, daß Simmel mit diesem Werk bewußt die Beschränktheiten der Schulphilosophie seiner Zeit zugunsten einer Hinwendung zu realen Sachverhalten durchbrochen und damit eine neue Art der Wirklichkeitsanalyse praktiziert hat, die ihrerseits einen umfassenden philosophischen Erkenntnisanspruch verfolgt. Zum anderen stellt die Philosophie des Geldes eine Alternative zu einer rein "materialistisch" verfahrenden Analyse der Geldwirtschaft dar, indem sie die dem Gebrauch des Geldes zugrundeliegenden psychischen Prozesse aufzudecken beabsichtigt und seine Auswirkungen auf die allgemeine Kultur zu bestimmen versucht.

Der erste, von Simmel als "analytisch" bezeichnete Teil, enthält eine ausführliche werttheoretische Fundierung des Geldes, die im Unterschied zur Tradition der Arbeitswertlehre den Wert eines Gutes nur als eine relative Größe geltend läßt, deren Umfang sich an dem Ausmaß des Opfers bemißt, das wir zur Erlangung eines Gutes zu bringen bereit sind. Der ökonomische Wert eines Gutes stellt dabei einen rein intersubjektiven Tatbestand dar, weil der Wert des "Opfers" bzw. des Konsumverzichts eines Menschen zugleich der Gegenstand des Begehrens eines anderen Menschen ist. Im Akt des Tauschens werden Simmel zufolge somit zwei verschiedene Begehrungsintensitäten miteinander verglichen und insofern aneinander gemessen. Dadurch entsteht zugleich der Eindruck, daß sich die getauschten Gegenstände ihren Wert jeweils gegenseitig zusprechen, ohne daß noch ein Bezug auf den tauschenden Menschen erforderlich wäre (S. 23 ff.). Dies ist zugleich der Ausgangspunkt eines Objektivationsprozesses des wirtschaftlichen Wertes, der im Geld sein adäquates gesellschaftliches Symbol gefunden hat und von Simmel als Urbild aller anderen Formen der Verselbständigung von an sich rein subjektiven bzw. intersubjektiven Sachverhalten zu eigengesetzlich verlaufenden Sozialprozessen betrachtet wird (S. 93 ff.). Simmels Philosophie des Geldes stellt insofern eine kulturtheoretisch gewendete Entfremdungstheorie dar, als sie die scheinbare Objektivität und Autonomie des Sozialen auf die ihm zugrundeliegenden seelischen Vorgänge und Wertungen zurückzuführen versucht. Zugleich zeigt sie auf, wie sich das Geld in den "Zweckreihen" der menschlichen Handlungsverkettungen vom ursprünglich bescheidenen Rang eines reinen Mittels allmählich selbst zu einem scheinbar absoluten Selbstzweck bzw. Endzweck entwickelt hat (S. 254 ff.). Im "synthetischen" Teil stellt Simmel die Auswirkungen der Geldwirtschaft auf die verschiedensten Lebensbereiche innerhalb der modernen Kultur dar, wobei er zum einen den engen historischen Zusammenhang zwischen der Entfaltung der Geldwirtschaft und der individuellen Freiheit innerhalb der europäischen Neuzeit verdeutlicht und zum anderen verschiedene Formen der Monetarisierung von ursprünglich rein personalen Werten analysiert (S. 375 ff. u. 482 ff.). Das mit der Überschrift "Der Stil des Lebens" versehene letzte Kapitel stellt dabei den nicht nur für seine soziologische Wirkungsgeschichte bedeutendsten Teil dieses monumentalen Werkes dar. Hier zeigt Simmel zum einen, wie die Entfaltung der Geldwirtschaft mit einer zunehmenden Rationalisierung und Intellektualisierung aller übrigen Lebensbereiche im Sinne einer Steigerung der Rechenhaftigkeit und Berechenbarkeit aller Dinge einhergeht (591 ff.). Zum anderen legt Simmel hier erstmals ausführlich die Grundzüge seiner Kulturtheorie dar; ihm zufolge treten die objektive Kultur bzw. die "Kultur der Dinge" und die subjektive Kultur, d.h. die Kultur des Menschen zunehmend auseinander, weil der einzelne Mensch aufgrund der fortschreitenden gesellschaftlichen Differenzierung und Arbeitsteilung immer weniger in 
der Lage ist, sich in dem gesamtgesellschaftlich erreichten Stand der Kulturentwicklung persönlich wiederzuerkennen und diesen für seine eigene Lebensführung fruchtbar zu machen (617 ff.). Und schließlich weist Simmel nach, daß die Kategorien, welche das moderne Weltbild kennzeichnen, trotz ihres relativen bzw. rein relationalen Charakters genauso umfassend sind, wie dies einstmals auch für ein absolutistisches Weltbild der Fall war und daß das Geld deshalb das adäquateste historische Symbol für den allgemeinen Relativitätscharakter des Seins darstellt (S. 655 ff.).

Obgleich Simmel selbst nicht schulbildend wirkte, hat seine Philosophie des Geldes der Kulturphilosophie und kulturwissenschaftlichen Forschung des 20. Jahrhunderts dennoch wichtige Anstöße gegeben. Ihr ist es zu verdanken, daß die Marxismus-Diskussion seit der Jahrhundertwende eine philosophische Tiefenschärfe anzunehmen begann, die in Georg Lukács' Buch "Geschichte und Klassenbewußtsein" ihren ersten Höhepunkt fand und sich auch in den kulturtheoretischen Schriften von namhaften Vertretern der Kritischen Theorie vorteilhaft niederschlug. Simmels eigene "Zeitphilosophie", wie er sie in der Philosophie des Geldes entfaltet hatte, stellt aber nicht nur die verborgene theoretische Klammer eines im Laufe des 20. Jahrhunderts gänzlich in zahlreiche Einzelströmungen auseinandergefallenen Kulturdiskurses dar, sondern zugleich einen sublimen Dialog mit der gesamten philosophischen Tradition des Abendlandes, die bei ihm ihren vorläufigen Abschluß fand, bis Martin Heidegger erneut den Versuch unternahm, die Vollendung der abendländischen Metaphysik durch den neuerlichen Rückgriff auf die uralte, bereits von den Vorsokratikern gestellte "Seinsfrage" zu überbieten, auf die Simmel mit seinem Versuch eines "modernen Heraklitismus" seinerseits eine zeitgemäße Antwort zu geben versuchte. Simmels Philosophische des Geldes hat aber auch der deutschen Tradition der Kultursoziologie entscheidende Impulse bei ihrem Bemühen gegeben, gewisse Einseitigkeiten der materialistischen Geschichtsbetrachtung durch eine Hinwendung zu rein kulturell bedingten Sachverhalten zu überwinden. Insbesondere sein Bestreben, die Kapitalismusproblematik nicht einseitig auf eine Geschichte von Klassenkämpfen zu reduzieren, sondern die intimen Verwandtschaften zwischen einer voll entfalteten Geldwirtschaft und anderen Erscheinungsformen des okzidentalen Rationalismus aufzuspüren, hat seinen Niederschlag in den entsprechenden Arbeiten von Werner Sombart, Max Weber und Max Scheler gefunden. Die von ihm aufgeworfene Relativismusproblematik ist ferner in der von Karl Mannheim entwickelten Variante der modernen Wissenssoziologie wieder aufgegriffen und produktiv weiterentwickelt worden. Und seine Analyse der Vergesellschaftungsfunktion von Austauschprozessen ist später von George Caspar Homans und Peter Blau zur Grundlage der modernen soziologischen Austauschtheorie gemacht worden.

Literatur: Frisby, D.: Fragmente der Moderne. Georg Simmel - Siegfried Kracauer - Walter Benjamin, RhedaWiedenbrück: Daedalus 1989; Kintzelé, J./Schneider, P. (Hrsg.): Georg Simmels Philosophie des Geldes, Frankfurt am Main: Hain 1993; Flotow, P. v., Geld, Wirtschaft und Gesellschaft. Georg Simmels Philosophie des Geldes, Frankfurt am Main: Suhrkamp 1995; Lichtblau, K.: Georg Simmel, Frankfurt am Main/New York: Campus 1997.

Klaus Lichtblau

In: Sven Papcke / Georg W. Oesterdieckhoff (Hrsg.), Schlüsselwerke der Soziologie, Wiesbaden: Westdeutscher Verlag 2001, S. 447-449.

(C) 2001-2003 Fachbereich Gesellschaftswissenschaften, Johann Wolfgang Goethe-Universität Frankfurt/Main 